\title{
Review: minimally invasive strabismus surgery
}

\begin{abstract}
This article reviews the principles and different techniques used to perform minimally invasive strabismus surgery (MISS). This term is used for strabismus surgeries minimizing tissue disruption. Muscles are not accessed through one large opening, but using several keyhole openings placed where needed for the surgical steps. If necessary, tunnels are created between cuts, which will allow performing additional surgical steps. To keep the keyhole openings small, transconjunctival suturing techniques are used. The cuts are always placed as far away from the limbus as feasible. This will reduce the risk for postoperative corneal complications and it will ensure that all cuts will be covered by the eyelids, minimizing postoperative visibility of surgery and patient discomfort. Benefits from minimizing anatomical disruption between the muscle and the surrounding tissue are a better preservation of muscle function, less swelling, and pain, and more ease to perform reoperations. MISS openings allow to perform all types of strabismus surgeries, namely rectus muscle recessions, resections, plications, reoperations, retroequatorial myopexias, transpositions, oblique muscle recessions, or plications, and adjustable sutures, even in the presence of restricted motility.
\end{abstract}

Eye (2015) 29, 225-233; doi:10.1038/eye.2014.281; published online 28 November 2014

Minimally invasive approaches to strabismus surgery decrease tissue traumatism, postoperative complications, patient discomfort, hospital stay, and working disability. Tissue traumatism depends on the number and types of muscles operated on, the muscle surgery technique itself, and the type and location of the conjunctival openings. For rectus muscles,
DS Mojon ${ }^{1,2,3}$

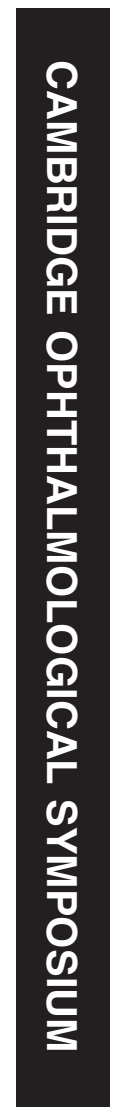

many surgeons use Harms' limbal opening (Figure 1a) over a quadrant, ${ }^{1}$ which has been popularized by von Noorden. ${ }^{2,3}$ It permits a full visualization of the operated muscle and avoids all the problems like strong muscle scarring or bleeding occurring with openings over the muscle bulk. If used for horizontal rectus muscle surgery, patients will experience considerable postoperative discomfort and the interpalpebral conjunctiva will be red for a couple of weeks. Harms' opening will be less visible and induce less discomfort if used for vertical rectus muscles. Horizontal limbal openings are prone for corneal dellen formation and Tenons capsule prolapse. Parks' introduced a fornix conjunctival approach for rectus muscle surgery (Figure 1b). Such openings remain covered by the lids. ${ }^{4}$ Parks' technique decreases markedly the postoperative discomfort.

However, the technique is difficult to use in children because prominent Tenons capsule protruding from the cuts may decrease visibility, in cases with significant preexisting scarring, and in older patients with inelastic conjunctiva, which might tear. Park's technique does not significantly reduce the area of anatomical disruption between the muscle and perimuscular tissue compared with Harms' opening. Usually, there is a need for an assistant surgeon to displace the opening over the operated muscle.

Gobin described the principle of access for rectus muscles through two small radial openings, one along the superior and the other along the inferior muscle margin (Figure 1c) to perform hang-back recessions. ${ }^{5}$ This principle of access has been adapted and developed further to allow to perform all types of strabismus surgeries, namely rectus muscle recessions, resections, plications, reoperations, retroequatorial myopexias, transpositions, oblique muscle recessions and plications, and adjustable sutures. ${ }^{6,7}$ For strabismus surgeries performed through keyhole openings, the term
${ }^{1}$ University of Bern, Bern, Switzerland

${ }^{2}$ Department of Ophthalmology, Allgemeines Krankenhaus, Linz, Austria

${ }^{3}$ Airport Medical Center Eye Clinic, Zürich, Switzerland

Correspondence: DS Mojon, Airport Medical Center Eye Clinic, Prime Center 1, PO Box 2128, 8060 Zürich, Switzerland Tel: +41 43816 7000; Fax: +4143816 7002 E-mail: daniel.mojon@ gmail.com

Received: 6 October 2014 Accepted: 15 October 2014 Published online: 28 November 2014 
a

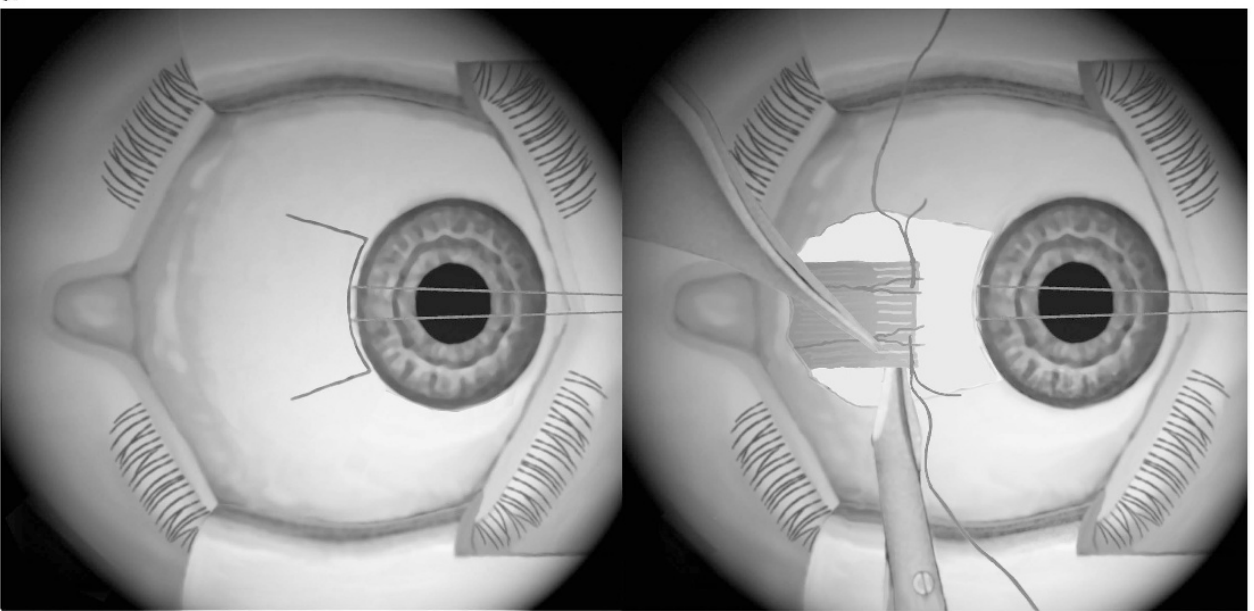

b

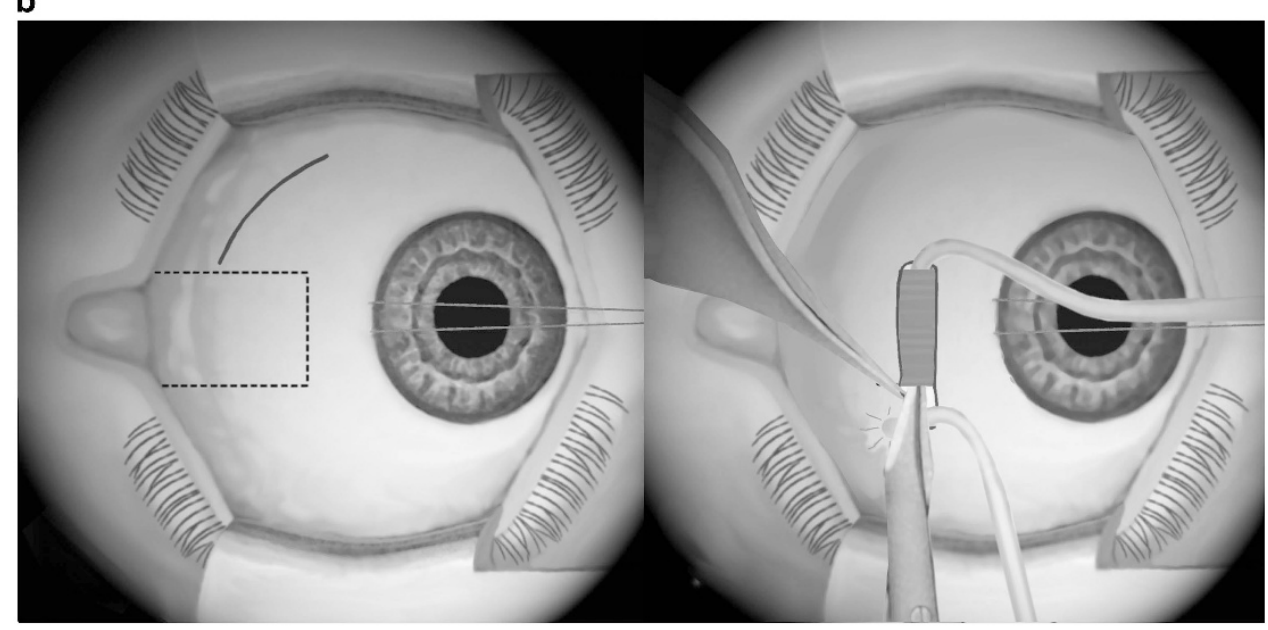

c

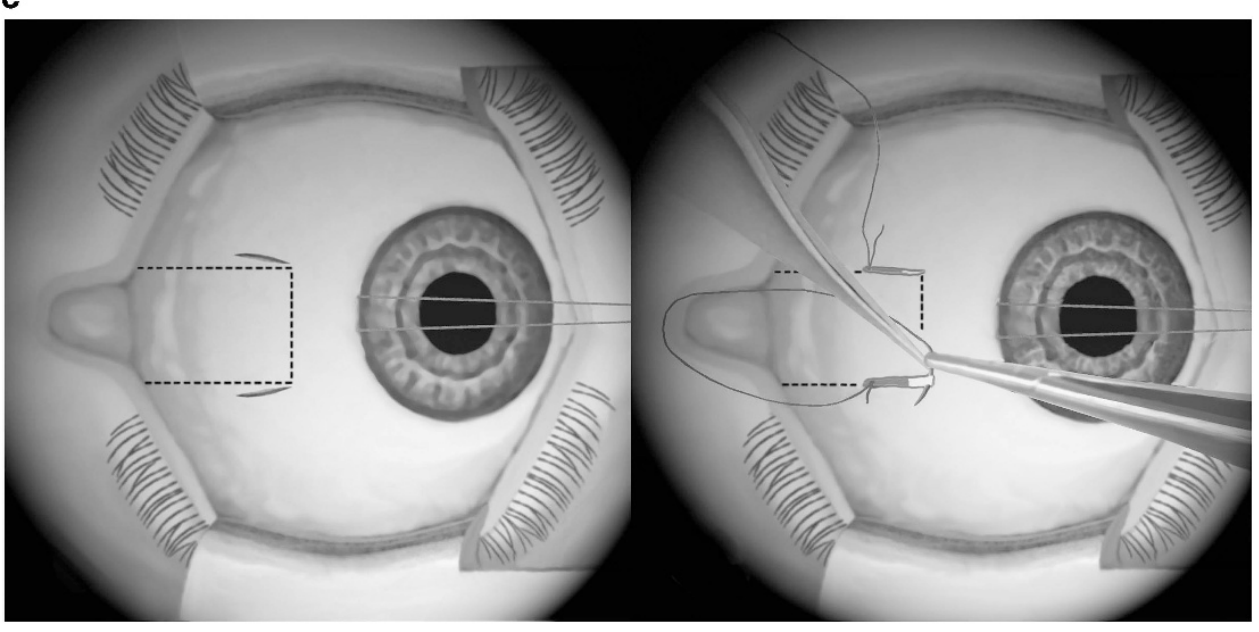

Figure 1 Types of conjunctival openings for strabismus surgery. (a) Harms' limbal opening allows an optimal visualization of the operating site. If used for horizontal rectus muscle surgery, it will induce considerable postoperative patient discomfort and a long lasting eye redness and lid swelling. (b) Parks' fornix conjunctival approach is much less invasive than Harms' limbal opening. The opening in the fornix is displaced over the muscle in order to perform a muscle weakening or strengthening. This opening will usually only allow to perform primary horizontal muscle surgeries and repeat surgeries with little scarring. (c) MISS conjunctival openings are placed far away from the limbus in a way to allow optimal visualization of all important surgical steps. MISS techniques can be used for all types of eye muscle surgery including complex repeat surgeries and transposition surgeries. 
minimally invasive strabismus surgery (MISS) has been introduced. ${ }^{6-8}$ Table 1 summarized the principles of MISS. Disadvantages of MISS include a possibly increased risk for scleral perforations and the rather long learning curve. The use of the microscope is advocated for MISS in order to allow an optimal visualization and to avoid complications. Instead of one large opening, several small cuts are placed where the main surgical steps, usually suturing, have to be performed. Openings are placed as far away from the limbus as possible to minimize postoperative discomfort. For several surgical procedures it is necessary to create tunnels. They are always needed between the two parainsertional cuts to isolate the muscle insertion before recessing or advancing a muscle. In addition, they may be necessary to access more posterior parts of the muscle or perimuscular tissue not lying below the opening. If necessary, cuts can be joined to a larger opening. For example, for rectus surgery, the two parainsertional cuts can be prolonged anteriorly and joined at the limbus in order to achieve Harms limbal opening. Perimuscular tissue dissection is reduced to the absolute minimum, which will still allow to displace or anchor the muscle. Usually, a more extensive perimuscular tissue dissecton cannot be avoided in repeat surgery to avoid a cosmetically disturbing displacement of the conjunctiva, Teneon's tissue, and orbital fat. Postoperatively, the MISS openings should be all covered by the eyelids minimizing visibility of surgery and patient discomfort. MISS openings markedly reduce the frequency and severity of corneal complications, for example, dry eye and dellen formation, and will allow wearing contact lenses earlier. Long-term benefits are avoidance of an increase of redness of the visible conjunctiva and a decreased scarring of the perimuscular tissue, which will facilitate reoperations. MISS preserves most perilimbal episcleral vessels ${ }^{9}$ and, therefore, may reduce the risk for anterior segment ischemia. ${ }^{9}$ As MISS also significantly reduces the anatomical disruption between the muscle and the surrounding tissue, reoperations will be easier. Table 2 shows a comparison of the three most frequently used access techniques for strabismus surgery.

\section{Visualization of the operating site while performing MISS}

Although it is advisable to use the operating microscope, MISS can also be performed using magnifying spectacles. However, a higher magnification allows smoother tissue dissection, easier detection of anatomical abnormalities, and better control of bleeding.

\section{Muscle exposure and wound closure for MISS}

The operated muscle is exposed by one prolene 6-0 limbal traction suture, allowing the performance of MISS without an assistant surgeon. Special care has to be taken when operating on the medial rectus muscle because the traction suture may touch the cornea, which may cause a corneal erosion. If the globe can be insufficiently rotated, consider performing two additional parainsertional scleral traction sutures after performing the two small

Table 1 The concept of MISS consists of the following principles

Use of a microscope

Use of corneal and, if necessary, more posterior traction sutures

Avoidance of conjunctival cuts not necessary to perform surgical maneuvers

Placement of conjunctival openings as far away from the limbus as possible

Performance of all feasible surgical steps through tunnels

Reduction of total conjunctival opening size by using several keyhole openings instead of one large access

Reduction number and size of conjunctival openings by using transconjunctival suturing techniques

Choose location of keyhole cuts allowing to join them if increased visibility is needed

Minimization of perimuscular tissue disruption

Table 2 Comparison of Harms' limbal opening, Parks' fornix technique and MISS

\begin{tabular}{llll}
\hline Type of surgery & Limbal & Fornix & MISS \\
\hline Difficulty to perform & medium & difficult & $\begin{array}{l}\text { Plications: medium } \\
\text { Recessions: difficult }\end{array}$ \\
& & $\begin{array}{l}\text { TRASU, revisions, transpositions, faden: difficult to very difficult } \\
\text { Formation of dellen }\end{array}$ & Rather frequent \\
Postopreative discomfort & Strong & Very rare & Very rare \\
Postopreative visibility & Strong & Minimal \\
Ease to perform repeat surgery & Moderate & Modium & Minimal \\
\hline
\end{tabular}

Abbreviations: MISS, minimally invasive strabismus surgery; TRASU, transconjunctival suturing 
conjunctival insertions. Traction on such sutures will retract the globe into the orbit and usually allow even the operate on already considerably recessed, and still severely restricted rectus muscles, for example, in patients with thyroid eye disease. The scrub nurse may need to hold temporarily a spatula or a hook, especially for repeat or superior oblique muscle surgery. Although some surgeons performing MISS do not close the keyhole openings at the end of surgery, I believe that all openings should be closed because leaving them open might increase the risk of infections. I use single, resorbable 8-0 sutures. As the cuts are far away from the limbus, suturing will usually not induce disturbing foreign body sensation.

\section{MISS for primary rectus muscle weakening and strenghtening}

Two radial keyhole parainsertional cuts are performed.6,10,11 For correct cut placement, use the muscle vessels as a landmark. To better distinguish between conjunctival and muscle vessels, the eye can be cyclorotated using the traction suture. If hardly visible, try to locate the adjacent rectus muscles, which will allow extrapolating the position of the operated muscle. If the planned opening might lie over the muscle, care should be taken to avoid cutting large vessels, which may lead to a hemorrhage making further surgery through the small opening difficult. Patients with reduced elasticity of the conjunctival tissue require larger cuts in order to avoid conjunctival tearing while using instruments. For recessions, after identifying the borders of the rectus muscle, a tunnel is created between the two cuts separating the conjunctiva and Tenons capsule from the muscle. The first muscle border is hooked using a small hook. A suture is applied through the lateral third of the rectus muscle tendon as close as possible to the insertion (Figure 2a, left). If prominent vessels are visible at the insertion site, they should be cauterized before placing the sutures. This procedure is repeated after introducing the hook through the other keyhole opening. Then, the tendon is detached (Figure 2a, middle). In order to avoid a tearing of the insertion or to cut a suture, relaxing incisions should be performed at both insertion borders before complete detachment of the remaining central part. After measurement of the amount of recession, the

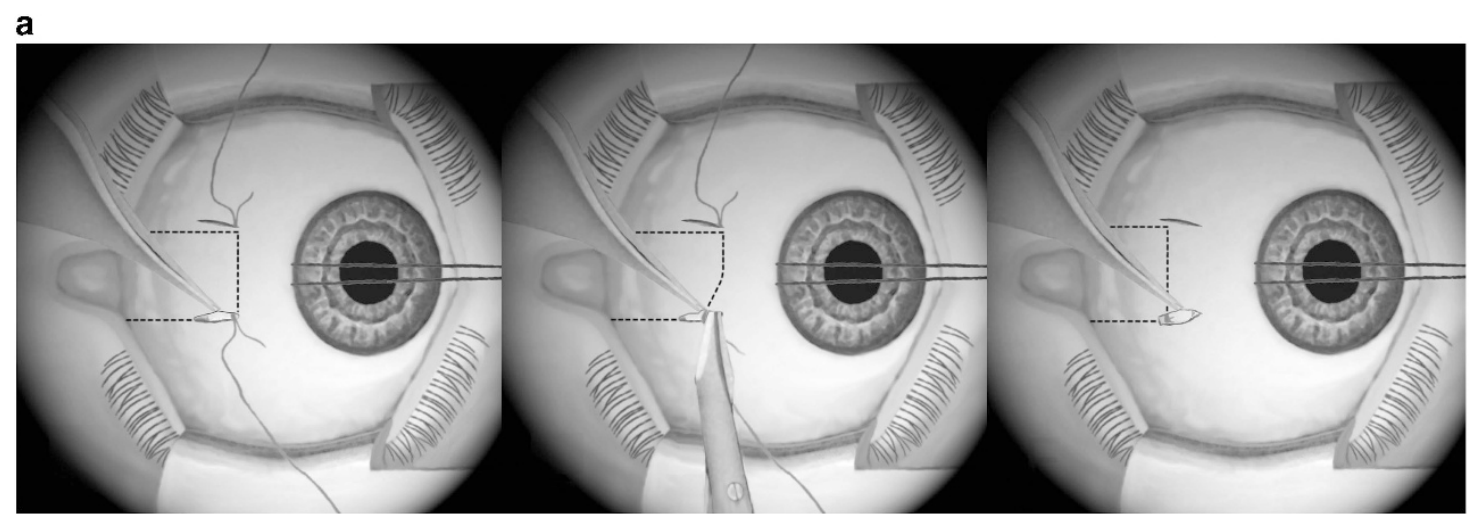

b

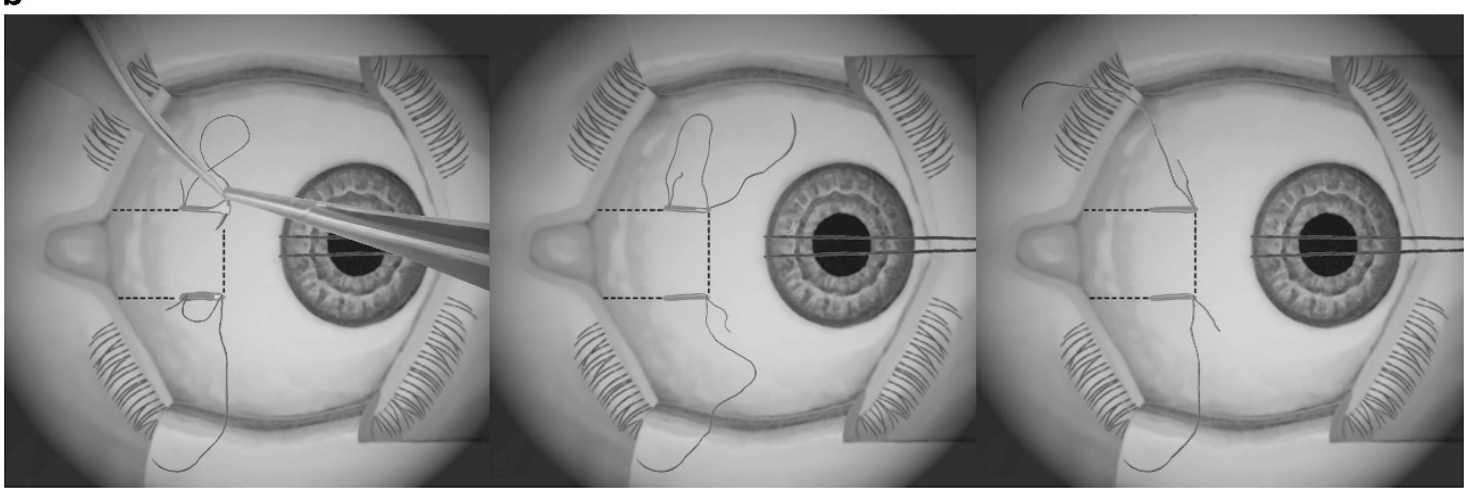

Figure 2 (a) MISS rectus muscle recession. Left: the muscle is sutured at the original inserteion. Middle: the muscle is desinterted. Right: the muscle is reattached after measuring the recession distance with a caliper. (b) MISS marginal dissection rectus muscle placation. Left: the muscle is sutured after measuring the plication distance. The first suture is passed through the original insertion. Middle: the second suture is passed through the original insertion. Right: the muscle is plicated by pulling the two sutures without using a spatula. 
tendon is reattached with the two sutures to the sclera (Figure 2a, right). Alternatively, the muscle reattachment sutures can be pre-placed before the muscle desinsertion. This may avoid that scleral suturing has to be performed under suboptimal visibility because of hemorrhage. The disadvantage is that there is an increased risk to cut the pre-placed sutures during the muscle desinsertion. To perform a rectus muscle resection, a tunnel is created after applying two parainsertional cuts. For a plication, there is no need for a tunnel because a marginal dissection of the rectus muscle is sufficient. ${ }^{12}$ After identifying the borders of the muscle, it is hooked. Two sutures are applied to the upper and lower borders of the muscle at a distance from the tendon insertion site that corresponds to the reinforcement amount. If at this location prominent vessels are visible, they should be cauterized before suturing. Next, the sutures are passed at the physiologic borders of the tendon insertion (Figure $2 b$, left). The muscle is plicated by tying the two sutures (Figure $2 b$, middle and right). For recessions, the muscle between the insertion and the sutures is excised and discarded before advancing the remaining muscle.

Plications have several advantages. Surgery is faster and less invasive and, thus, postoperative discomfort and visibility is reduced. In case of overcorrection, the fold can usually be opened in the first couple of weeks without tissue loss. The folds allow to easily perform adjustable surgery. Usually, in primary position, MISS openings will not be visible. The cuts can be visualized on side gaze by pulling on the eyelids.

\section{MISS TRASU}

MISS transconjunctival suturing (TRASU) is a technique that allows to perform all types of the muscle and the sclera suturing through a keyhole opening without significantly displacing it. Such a suturing technique minimizes conjunctival opening size and tissue traumatism. The first TRASU technique that has been described was introduced for rectus muscle recessions. ${ }^{11}$ It allows to perform large recessions and to reattach a muscle without need to enlarge the cut as posterior as the new the insertion site lays. The suture is performed by observing the scleral passage through the keyhole cut. In order to be able to pass the needle completely through the sclera, the needle has to exit through the overlying Tenons capsule and conjunctiva. Then, the needle is retrieved by pulling backwards on the suture allowing the surgeon to place a standard knot. In another technique, (Figure $3 a$ and $b$ ) the needle is only passed partially through the conjunctiva and is retrieved as soon as the needle passed the muscle (if a muscle is sutured) or passed the sclera (if a scleral reattachment is prepared).

\section{MISS for rectus muscle posterior fixation suture}

For access, two small L-shaped cuts are performed slightly anterior to location where the scleromuscular sutures will be placed. A more anterior prolongation of the cuts will allow combining the posterior fixation sutures with a recession or plication of the same rectus muscle. With blunt scissors the episcleral tissue is separated from the muscle sheath and the sclera. With a measure caliper the location of the scleromuscular sutures is marked. Then, on each muscle border a nonresorbable suture is passed through the sclera and onethird of the muscle (Figure 4, left). Tightening is performed by a three-throw adaptation suture followed by two securing loops. ${ }^{13}$

\section{MISS for rectus muscle transposition}

We avoid full transpositions of rectus muscles because partial transpositions will also allow correcting large deviation. Transposition of half of the adjacent rectus muscles is performed using four keyhole MSS openings. For larger deviations, the split tendons need to undercross the weak rectus muscle. For very large deviation, the ipsilateral antagonist of the weak muscle needs to be recessed. The rectus muscles adjacent to the weak muscle are split in the middle over a distance of $15 \mathrm{~mm}$. After applying two single sutures to each half muscle tendon, which will be transposed, they are desinserted. Now, the weak rectus muscle is exposed through two small parainsertional keyhole cuts. A blunt $20 \mathrm{G}$ sub-Tenon's anesthesia cannula is used to safely displace the needles of the sutures through the tunnels. After scleral anchoring, the half muscles are transposed by gently pulling the sutures (Figure 4, middle). ${ }^{14}$

After MISS transpositions on the first postoperative day, the perilimbal region is only mildly red and swollen.

\section{MISS for rectus muscle repeat surgery}

MISS for muscle repeat surgery is especially valuable because it avoids reopening of the scarred perilimbal conjunctiva. The principles of surgery for repeat rectus muscle recessions and repeat rectus muscle plications are identical to primary MISS surgery. Previously recessed muscles should always be advanced and not plicated. ${ }^{15}$

\section{MISS for oblique muscle recessions}

MISS inferior and superior oblique recessions of $<6 \mathrm{~mm}$ are performed using one opening over the muscle insertion. For larger recessions, two keyhole openings are 
.
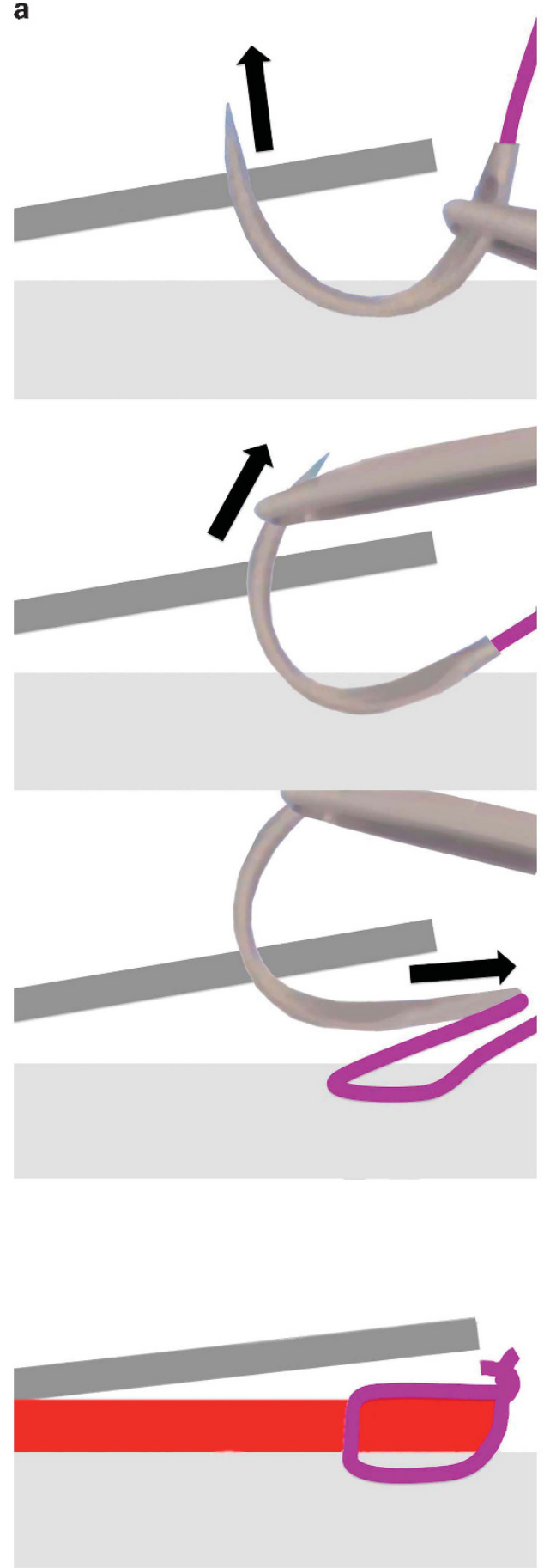

b
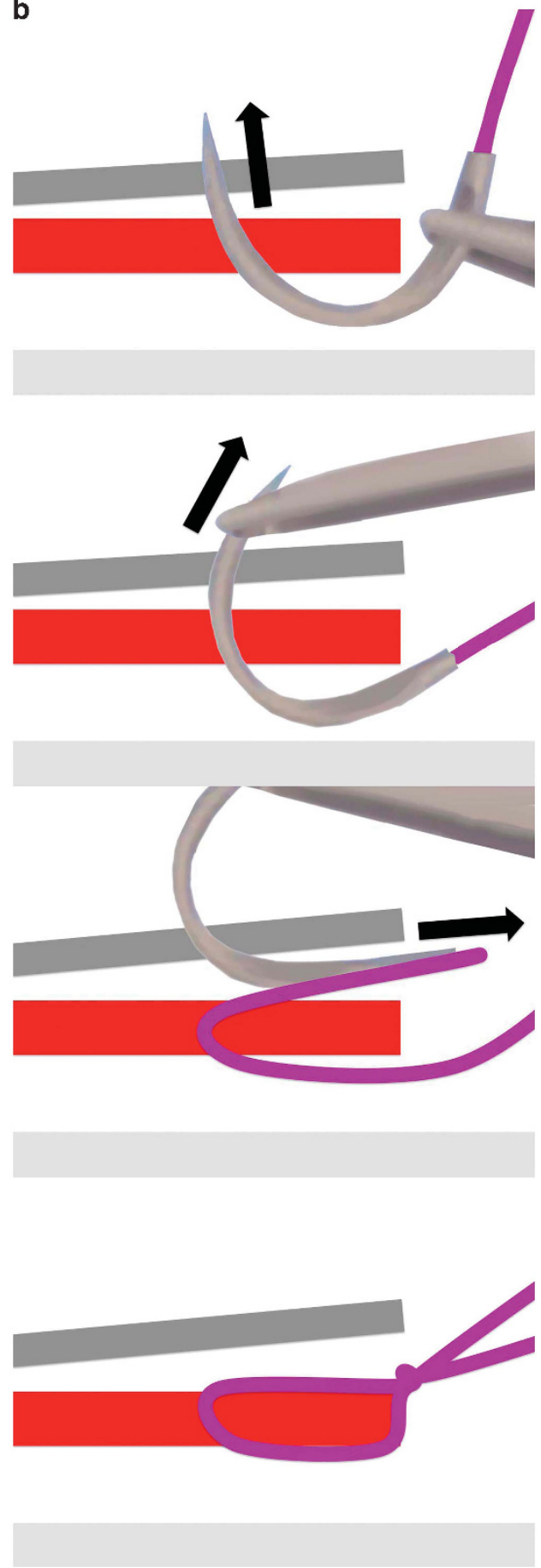

Figure 3 (a) Transconcuntival suturing to reattach the muscle to the sclera. After passing the tip of the needle through the keyhole opening, the sclera, and the conjunctiva, the needle tip is grasped with the needle holder from above the conjunctiva and retrieved partially until the whole needle has passed the sclera. Now, the needle is pulled back through the keyhole opening in order to reattach the muscle to the slera. (b) Transconcuntival suturing of a muscle. After passing the tip of the needle through the keyhole opening, the muscle, and the conjunctiva, the needle tip is grasped with the needle holder from above the conjunctiva and retrieved partially until the whole needle has passed the muscle. Now, the needle is pulled back through the keyhole opening in order to perform the muscle suture. 


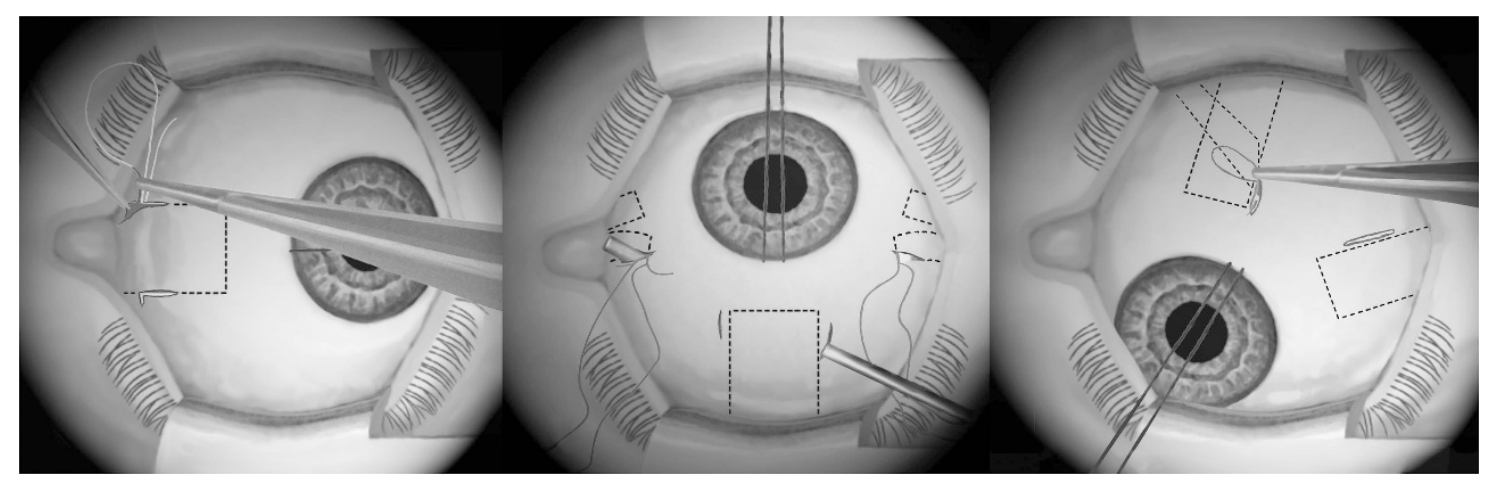

Figure 4 Advanced MISS techniques: retroequatorial myopiexia (left), muscle transpositions (middle), and oblique muscle recessions (right).

applied. The first one is used to desinsert the muscle insertion and the second one is used for the scleral anchoring (Figure 4, right). In order to safely pass the needle between the two openings, a blunt $20 \mathrm{G}$ subTenon's anesthesia cannula is used. ${ }^{16}$ MISS inferior and superior oblique muscle plications are performed through one L-shaped opening over the insertion. ${ }^{6}$

\section{MISS for fibrotic rectus muscle recessions}

In severely fibrotic muscles like in Duane retraction syndrome or thyroid eye disease, usually, scleral sutures cannot be applied before the eye can be adequately turned away from the field of surgery. Therefore, scleral reanchoring can only be performed after muscle desinsertion. ${ }^{6}$ If suturing seems to be dangerous, a loop recession should be considered.

\section{Adjustable sutures MISS}

MISS allows performing adjustable suture surgery as with open surgery. The adjustable sutures have to be placed in a manner that they become visible after removal of the conjunctival sutures, if an adjustment becomes necessary (Figure 5). An adjustment will not increase the postoperative discomfort and visibility of surgery. After an adjustment, the conjunctiva needs only to be closed if the adjustable sutures protrude through the openings. Thus, usually, an adjustment can be performed at the slit lamp.

\section{Specific complications of MISS}

The keyhole cuts may tear in older patients if they are excessively displaced with instruments. Tears not involving Tenons capsule will remain without consequences. If the tear involves Tenons capsule, a visible scar may result. Tears occurring over the muscle may induce scar tissue between the muscle and the

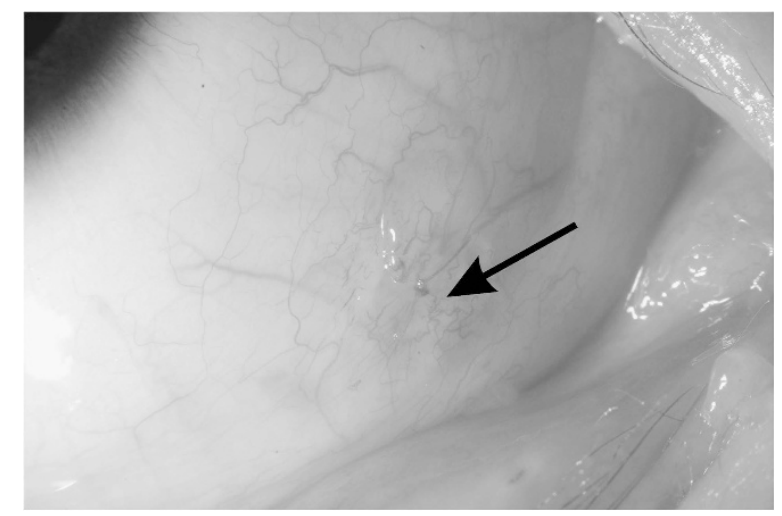

Figure 5 Appearance of the eye on the first postoperative day after adjustable suture surgery on a rectus muscle. The arrow indicates where the adjustable subconjunctival suture would lie in case an adjustment becomes necessary.

conjunctiva difficult to dissect if repeat surgery becomes necessary. Muscle desinsertion through a tunnel is more demanding. If the cut is too close to the sclera, a permanently visible bluish line may result, if too far away, the remaining tendon may visibly elevate the conjunctiva. An excessive bleeding that cannot be stopped makes an enlargement of the cuts necessary to cauterize the vessel. Usually, a conversion to a limbal opening can be avoided. Blood caught in the tunnels can be rinsed with BSS. Rinsing should be performed gently in order to avoid swelling of Tenons capsule. Larger bleedings into Tenons capsule shoud be excised.

Relatively, flat bleedings anteriorly to the insertion can be pushed back with a strabismus hook. Gently exert transconjunctivally a pressure on the sclera near the limbus, and move the hook more posteriorly until the blood clears through the small cuts. ${ }^{6}$

\section{How to start performing MISS}

Surgeons who want to switch from open surgery performed with magnifying glasses to MISS with the 
operating microscope should first only switch to the operating microscope. After feeling at ease with the microscope, switch to MISS. Start with primary horizontal rectus muscle displacements of $4 \mathrm{~mm}$ or less. Patients should be aged between 14 and 40 years. The thick Tenons capsule will make surgery more difficult in younger patients; reduced elasticity of the conjunctiva increases the risk for a conjunctival tear in older patients while introducing or manipulation instruments through the keyhole openings. ${ }^{6}$ The ideal before starting with a new technique is to personally visit an already skilled surgeon. I always welcome colleagues to come in the operating theater and assist MISS.

\section{Instruments for MISS}

In general, small instruments will help to avoid conjunctival tearing and allow a better visualization through the keyhole openings. The following instruments are suggested for MISS. Corneal rubbing of a medial traction suture can be prevented by clamping a long serrefine to the temporal border of to the eyelid speculum, which will act as a hypomochlion. A colibrie forceps with interdigitating teeth is used to fix the conjunctiva while performing the small openings and to stabilize the eye for traction and scleral anchoring sutures. A small, curved needle holder is ideal to apply sutures through MISS openings. A small, curved and blunt conjunctival scissor is used to cut the conjunctiva, the sutures, and the muscle and to bluntly dissect the muscle form its surrounding tissue. Curved forceps with serrated, less traumatizing tips should be used to hold the conjunctiva after performing the openings. Spatulas of different sizes are helpful to visualize the tissue through the cuts. Cauterization is best performed through the small openings with a bipolar, coaxial diathermy tip. A small strabismus hook will allow, if necessary, to introduce additional instruments through the same cut. A Castroviejo caliper is used to measure displacement distances. Pressure of its blunt tips for about $10 \mathrm{~s}$ against the sclera will leave a bluish indentation mark, which will be visible for up to $1 \mathrm{~min}$. This indentation technique works also transconjunctivally to perform TRASU as no dye is necessary. A $20 \mathrm{G}$ sub-Tenon's anesthesia cannula is helpful to safely displace needles through tunnels when rectus muscles are transposed or for larger, graded oblique muscle recessions. ${ }^{6}$

\section{Duration of MISS procedures}

Switching to MISS will initially increase your surgical time. However, after a while surgical times will become shorter compared with open surgery. Especially the marginal dissection technique ${ }^{12}$ allows to perform very quickly muscle reinforcements. Oblique muscle procedures will not be significantly faster. Conversely, rectus muscle transposition can be performed much faster because large openings are avoided. In order to avoid increasing excessively your total operating time on 1 day, start doing MISS only in one patient per day.

\section{Changes of dose-response relationships when switching to MISS}

If a surgeon performs MISS muscle displacements in the same way he was doing them with open surgery and if the amount of displacement is measured as usual, it is unlikely that dose-response relationships will markedly differ after switching operating technique. However, most probably it is prudent if all surgeons switching to MISS look for changes in their dose-response relationships. ${ }^{6}$

\section{Summary}

Today, strabismus surgeons should use conjunctival openings as far away from the limbus as possible because the limbus is very susceptible to surgical trauma. Permanent damages may occur, especially to the perilimbal vessels and stem cells. Avoiding a limbal opening will also decrease postoperative visibility of the surgical procedure, patient discomfort, scarring around the operated muscles, and reduce hospital stay and working disability. Placing several keyhole openings far away from the limbus, performing surgery using tunnels and using TRASU techniques are the most important concepts of strabismus surgical techniques summarized under the term MISS. Also Parks' technique achieves the goal of avoiding a limbal opening. However, Parks' technique is difficult to use in patients with inelastic conjunctiva, can usually not be performed without an assistant surgeon and does not really minimize perimuscular tissue traumatism. The technique cannot be adapted to be used for as many surgical techniques as MISS allows. Before starting with MISS, it is suggested to visit an experienced MISS surgeon. The author of this article always welcomes colleagues in the operating theater.

\section{Conflict of interest}

The author declares no conflict of interest.

\section{References}

1 Harms H. Über Muskelvorlagerung. Klin Monatsbl Augenheilk 1949; 115: 319-324.

2 von Noorden GK. The limbal approach to surgery of the rectus muscles. Arch Ophthalmol 1968; 80(1): 94-97. 
3 von Noorden GK. Modification of the limbal approach to surgery of the rectus muscles. Arch Ophthalmol 1969; 82(3): 349-350.

4 Parks MP. Fornix incision for horizontal rectus muscle surgery. Am J Ophthalmol 1968; 65(6): 907-915.

5 Gobin MH, Bierlaagh JJM. Chirurgie horizontale et cycloverticale simultanée du strabisme. Centrum voor Strabologie: Anvers, Belgium; 1994.

6 Mojon DS. Minimally invasive strabismus surgery. In: Fine HI Mojon DS (eds). Minimally invasive ophthalmic surgery. Springer: Heidelberg, Germany; 2009, pp 123-152.

7 Mojon DS. Minimally invasive strabismus surgery. Br J Ophthalmol 2009; 93(6): 843-844.

8 Mojon DS. Comparison of a new, minimally invasive strabismus surgery technique with the usual limbal approach for rectus muscle recession and plication. Br J Ophthalmol 2007; 91(1): 76-82.

9 Kushner BJ. Comparison of a new, minimally invasive strabismus surgery technique with the usual limbal approach for rectus muscle recession and plication. Br J Ophthalmol 2007; 91(1): 5.
10 Pellanda N, Mojon DS. Minimally invasive strabismus surgery technique in horizontal rectus muscle surgery for esotropia. Ophthalmologica 2010; 224: 67-71.

11 Mojon DS. A new transconjunctival muscle reinsertion technique for minimally invasive strabismus surgery. J Pediatr Ophthalmol Strabismus 2010; 47: 292-296.

12 Mojon DS. A modified technique for rectus muscle plication in minimally invasive strabismus surgery. Ophthalmologica 2009; 224(4): 236-242.

13 Mojon DS. Minimally invasive strabismus surgery for rectus muscle posterior fixation. Ophthalmologica 2009; 223(2): 111-115.

14 Mojon DS. Minimally invasive strabismus surgery (MISS) for rectus muscle transpositions. Br J Ophthalmol 2009; 93(6): 747-753.

15 Mojon DS. Minimally invasive strabismus surgery for horizontal rectus muscle reoperations. Br J Ophthalmol 2008; 92(12): 1648-1652.

16 Mojon DS. Minimally invasive strabismus surgery (MISS) for inferior obliquus recession. Graefes Arch Clin Exp Ophthalmol 2009; 247(2): 261-265. 